\title{
Lobe-specific modulation of B16MET melanoma lung metastases by nephrilin peptide
}

\author{
Desmond D. Mascarenhas ${ }^{1,2}$, Puja Ravikumar ${ }^{3}$, Edward P. Amento ${ }^{3}$ \\ 'Mayflower Organization for Research \& Education, Sunnyvale, CA 94085, USA. \\ ${ }^{2}$ Transporin, Inc., Sunnyvale, CA 94085, USA. \\ ${ }^{3}$ Molecular Medicine Research Institute, Sunnyvale, CA 94085, USA.
}

Correspondence to: Desmond D. Mascarenhas, Ph.D., Mayflower Organization for Research \& Education, 428 Oakmead Pkwy, Sunnyvale, CA 94085, USA. E-mail: desmond@transporin.com

How to cite this article: Mascarenhas DD, Ravikumar P, Amento EP. Lobe-specific modulation of B16MET melanoma lung metastases by nephrilin peptide. J Cancer Metastasis Treat 2020;6:23. http://dx.doi.org/10.20517/2394-4722.2020.52

Received: 17 May 2020 First Decision: 24 Jun 2020 Revised: 24 Jun 2020 Accepted: 06 Jul 2020 Published: 30 Jul 2020

Academic Editor: Rafat A. Siddiqui Copy Editor: Cai-Hong Wang Production Editor: Jing Yu

\begin{abstract}
Aim: Nephrilin peptide modulates systemic immune responses to trauma in contexts characterized by simultaneous inflammation and immunosuppression. This study explores the possibility that nephrilin peptide may modulate lung metastasis, which also occurs in an environment of concurrent inflammation and immunosuppression.
\end{abstract}

Methods: B16MET melanoma cells were injected via the tail vein of mice and the development of lung metastases was recorded in animals treated with nephrilin peptide or vehicle by subcutaneous bolus injection daily for three weeks. In a separate experiment, nephrilin was administered by subcutaneous bolus injection for seven days to study the biodistribution of peptide and possible changes to plasma cytokine levels.

Results: Nephrilin significantly suppressed B16MET lung metastases. Suppression was more effective in deep lobes with the poorest access to circulation: accessory > inferior > middle > superior. In a separate biodistribution study in mice, nephrilin showed similar biodistribution levels in kidney, liver, brain, and left lung, but significantly higher accumulation in the lobes of the right lung in a gradient that matched its effectiveness in suppressing metastases (accessory $>$ inferior $>$ middle). The latter environments were also characterized by significantly higher local concentrations of succinate, a proxy for lower levels of oxygenation.

Conclusion: Nephrilin accumulates preferentially in the deep lobes of the right lung in mice and inhibits B16MET right lung metastases in a lobe-specific manner.

Cc) (i) The Author(s) 2020. Open Access This article is licensed under a Creative Commons Attribution 4.0 International License (https://creativecommons.org/licenses/by/4.0/), which permits unrestricted use, sharing, adaptation, distribution and reproduction in any medium or format, for any purpose, even commercially, as long as you give appropriate credit to the original author(s) and the source, provide a link to the Creative Commons license, and indicate if changes were made.

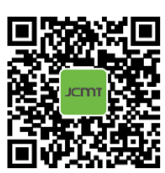


Keywords: Nephrilin, melanoma, metastases, deep lung, lobes, anoxia, oxidative stress, immune modulation

\section{INTRODUCTION}

The peptide nephrilin is an inhibitor of Rictor complex derived by fusing a 19 amino acid segment of Protor with the metal-binding domain of human insulin-like growth factor binding protein-3 (IGFBP3)-a 21 amino acid pro-homeostatic sequence that targets and preferentially enters stressed cells ${ }^{[1-2]}$.

Severe burn trauma is associated with secondary systemic effects including hyper inflammation, hypercatabolism, sepsis, organ failure, loss of glycemic control, delayed wound healing, and cognitive deficits $^{[3-8]}$. We have demonstrated the efficacy of nephrilin peptide in combating many of these pleiotropic effects in burn trauma as well as in several rodent models of metabolic and xenobiotic stress ${ }^{[9-14]}$. When nephrilin peptide is injected subcutaneously into rodents, its Protor sequence is believed to reduce oxidative stress in target tissues by modulation of Rac1 phosphorylation and, more speculatively, its IGFBP3 sequence may mediate homeostatic immunomodulatory functions in addition to its described transporter functions ${ }^{[12]}$. Nephrilin treatment following burn injury reverses epigenetic and signaling changes in kidney tissue that lead to the activation of Rac1, and lowers elevations in markers of systemic oxidative stress such as urinary 8-isoprostane and plasma OHDG ${ }^{[10-12]}$. Besides, an analysis of gene expression in the CNS after burn injury showed that nephrilin beneficially modulated the expression of genes associated with astrocytosis, oxidative stress, and immunosuppression ${ }^{[13]}$. Trauma is characterized by the co-existence of pro-inflammatory and immunosuppression phenomena, strikingly reminiscent of what has long been described for metastatic niche formation ${ }^{[15,16]}$. The role of oxidative stress and pro-fibrotic cytokines has been amply documented as instrumental in the sculpting of the latter environment(s). We asked whether nephrilin might modulate the latter environment, as we have shown that it has beneficial effects on the former.

Previous studies have shown that the lobes of the rodent right lung differ in their access to the arterial and lymphatic circulation, susceptibility to anoxia, and local concentrations of pro-inflammatory factors such as lung elastase ${ }^{[17]}$. We were interested in examining the biodistribution and effectiveness of nephrilin peptide in the lobes of the rodent lung, to gain a better understanding of how nephrilin accesses deep lung environments. This variable is relevant in critical care settings, particularly in the context of major comorbidities of sepsis and burn trauma, such as ARDS ${ }^{[18,19]}$. Nephrilin has shown beneficial effects in rodent models of sepsis and burn trauma, as noted above.

Finally, lung metastasis is accelerated by profibrotic and pro-metastatic factors such as CCL12 and TIMP- ${ }^{[20,21]}$. We examined the possible effects of nephrilin on the circulating levels of these factors.

\section{METHODS}

\section{Reagents}

Nephrilin peptide (1) and FITC-labelled tracer peptide, the IGFBP3-derived portion of the nephrilin peptide sequence (fitc-KKGFYKKKQCRPSKGRKRGFCW) were synthesized by Lifetein LLC (Hillsborough, NJ). Antibodies for ELISAs were purchased from Abcam (Cambridge, MA), and chemicals from Sigma-Aldrich (St. Louis, MO) unless otherwise specified.

\section{Murine models}

Animals were housed in clean cages on a $12 \mathrm{~h}$ light/dark cycle with access to standard chow and water ad libitum. Animals were allowed to acclimate for one week before the experiment. All animal procedures were performed in adherence to the National Institute of Health's Guide for Care and Use of Laboratory 
Animals and approved by the Institutional Animal Care and Use Committee (IACUC) of the Molecular Medicine Research Institute. At the end of the study, period animals were euthanized by decapitation as approved by MMRI IACUC guidelines, the NIH's Office of Laboratory Animal Welfare (OLAW), and AVMA recommendations.

For the lung metastasis study, 7-week old female $B_{6} D_{2} F_{1}$ mice (Charles River; 18-22 gram body weight) received implantation via tail vein of 1x10e5 B16MET-e100 tumor cells per animal. Beginning on Day 2, animals received either saline or nephrilin peptide $(1.2 \mathrm{mg} / \mathrm{kg})$ daily via subcutaneous bolus injection, for 3 weeks ( $n=6$ per group). At the end of the treatment period, animals were sacrificed and lung metastases in each lobe of the right lung were counted.

For biodistribution studies, 10-week old male C57BL6 mice (Jackson Laboratories; 30 grams body weight) were used. Animals received either saline or nephrilin peptide $(4 \mathrm{mg} / \mathrm{kg})+$ FITC-labelled peptide tracer (1:100 molar ratio to nephrilin) via subcutaneous bolus injection once daily for seven days $(n=3$ per group). The concentration of tracer peptide administered is too low to generate measurable biological effects, of itself. At the end of the treatment period, animals were sacrificed and left lung, left kidney, brain (left hemisphere), and liver (left half) were immediately stored in formalin for further histological analysis. Brain (right hemisphere), right kidney, liver (right half), plasma, and each lobe of the right lung were immediately frozen at $-80^{\circ} \mathrm{C}$ for further analysis.

\section{Plasma cytokine/chemokine analysis}

Analysis of plasma was performed using a mouse cytokine 44-plex immunoassay (Custom Plex Discovery Assay, Eve Technologies, Calgary, AB). Statistically significant differences (Student's $t$-test, $P<0.05$ ) between saline-treated and nephrilin-treated animals in the biodistribution experiment were found in the plasma levels of two of the 44 analytes tested.

\section{Tissue extracts}

Frozen tissue extracts were prepared using the CellLyticMT extraction buffer (Sigma-Aldrich, St. Louis, $\mathrm{MO}$ ) as specified by the manufacturer. Extracts were stored at -20 degrees $\mathrm{C}$ before assay by ELISA (for nephrilin peptide, using in-house polyclonal antibody \#2501) or for succinate assay using a kit purchased from Megazyme, Inc (Chicago, IL).

\section{Statistical analysis}

Data are presented as means \pm SD unless otherwise indicated. Probability values ( $P$ values) were computed using Student's $t$-test and expressed relative to the saline-treated group.

\section{RESULTS}

\section{Biodistribution of nephrilin}

Figure 1 shows tissue sections of the brain, kidney, left lung, and liver from mice injected with either saline or nephrilin $(4 \mathrm{mg} / \mathrm{kg})+1: 100$ FITC-tracer peptide (see methods). A fairly even distribution of peptide across tissues is visible in the slides, and corroborated by the biodistribution of nephrilin peptide in the brain (0.81 $\mu \mathrm{g}$ nephrilin/mg total tissue protein), kidney (0.97) and liver (0.74), as measured by ELISA. The concentration of nephrilin in the left lung was not measured, as that tissue was fixed in formalin. The concentration of nephrilin and tracer peptide in the right lung was measured. Table 1 shows the distribution of nephrilin in the four lobes of the right lung. There is a gradient of accumulation of peptide $(\mu \mathrm{g} / \mathrm{mg}$ total tissue protein) in the deep lobes: accessory $(5.07)>$ inferior $(3.45)>$ middle $(2.84)>$ superior (2.12). This gradient is reminiscent of that previously shown for lung elastase in rodent lung ${ }^{[17]}$. We also measured the concentration of succinic acid in tissue extracts of each lobe of the right lung. Interestingly, 


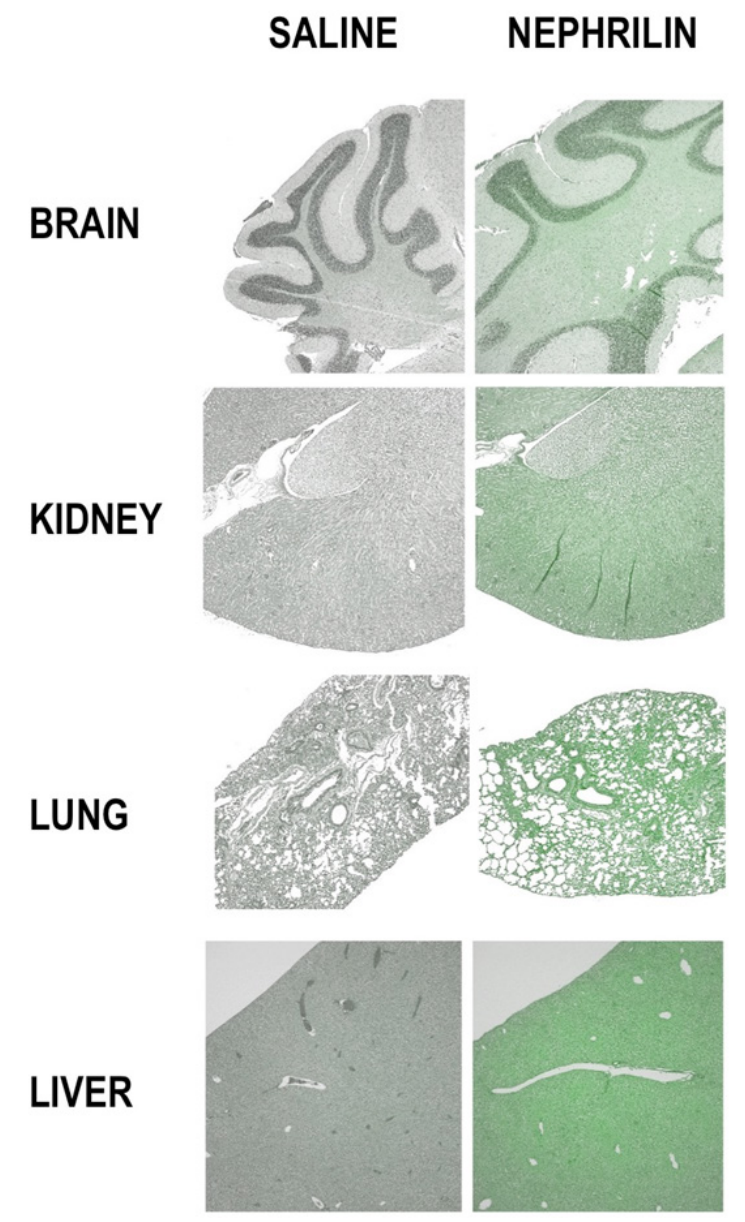

Figure 1. Biodistribution of tracer peptide in major organs. See text and Methods for a description of the tracer peptide and histological methods

Table 1. Biodistribution of nephrilin in right lung lobes ${ }^{[1]}$

\begin{tabular}{|c|c|c|c|c|}
\hline Treatment Group & Superior & Middle & Inferior & Accessory \\
\hline Saline-treated & $0.11 \pm 0.32$ & $0.19 \pm 0.22$ & $0.10 \pm 0.12$ & $0.10 \pm 0.41$ \\
\hline Nephrilin-treated & $0.12 \pm 0.37$ & $0.84 \pm 0.58$ & 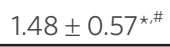 & $3.07 \pm 0.90^{\star \text { *\#\# }}$ \\
\hline
\end{tabular}

${ }^{[1]}$ Tissue nephrilin measured by ELISA ( $\mu$ g nephrilin/mg tissue protein); ${ }^{\star} P<0.05$ vs. saline-treated group; ${ }^{\#} P<0.05$ vs. superior lobe

the concentration of succinic acid was $22 \%$ higher $(P<0.001)$ in the accessory, inferior and middle lobes, when compared to the superior lobe, suggesting lower oxygenation levels in those tissues.

\section{Plasma cytokines}

Of the 44 cytokine/chemokine analytes measured in plasma, only two differed significantly between the mice treated with nephrilin versus those treated with saline: CCL12 (MCP-5) and TIMP-1 were both significantly lower in the nephrilin group [Figure 2]. Both CCL12 and TIMP-1 are believed to play a profibrotic role ${ }^{[20,21]}$ and fibrosis is canonically described as a feature of the tumor environment ${ }^{[2]}$.

\section{Lung metastases}

Table 2 shows B16MET melanoma lung metastases in the saline and nephrilin treated groups on Day 21 . The average number of metastases decreases along with the superior $>$ middle $>$ inferior $>$ accessory gradient, consistent with relative access to the circulatory system. However, the percent reduction in 


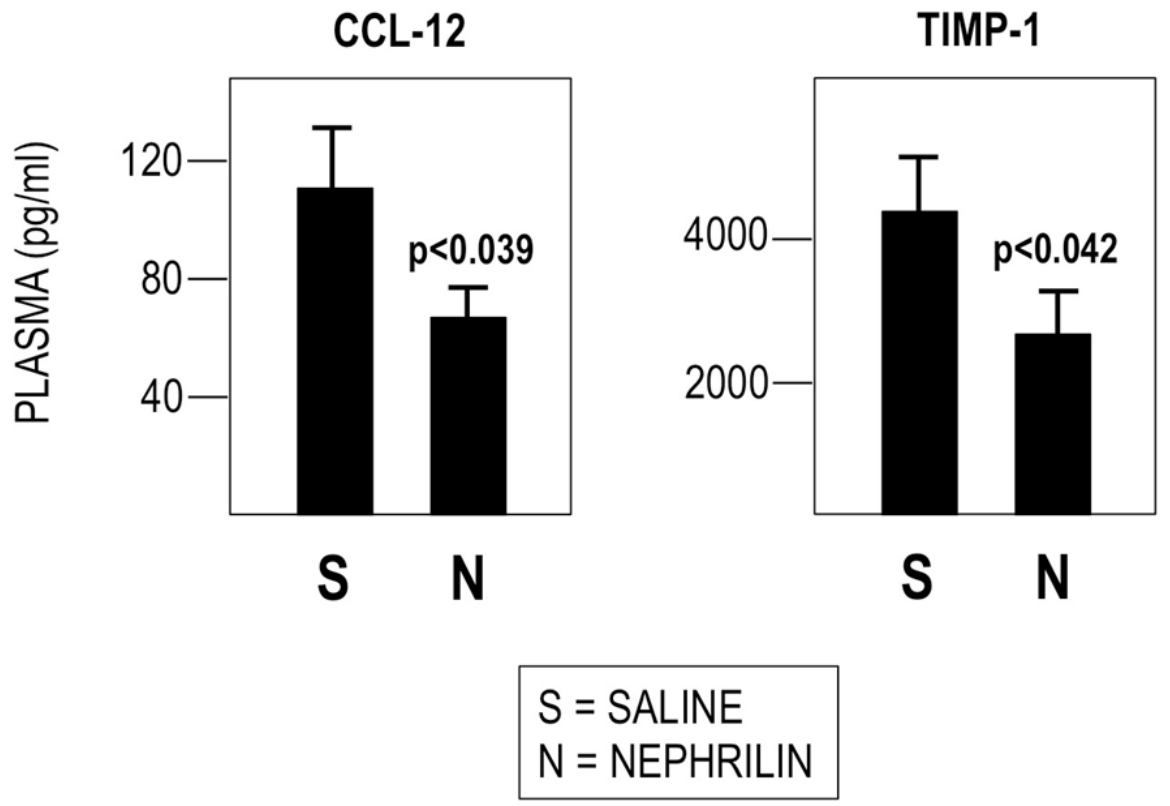

Figure 2. Plasma analytes in mice treated with saline or nephrilin for seven days

Table 2. B16MET melanoma metastases in lung lobes at 3 weeks post-injection ${ }^{[2]}$

\begin{tabular}{lcccc}
\hline Treatment Group & Superior & Middle & Inferior & Accessory \\
\hline Saline-treated & $17.83 \pm 3.43$ & $15.83 \pm 3.43$ & $14.83 \pm 2.64$ & $6.17 \pm 1.72$ \\
Nephrilin-treated & $17.33 \pm 3.33$ & $11.83 \pm 2.56^{\star}$ & $10.00 \pm 4.29^{\star}$ & $3.33 \pm 1.63^{\star}$ \\
\hline
\end{tabular}

${ }^{[2]}$ All lobes combined: saline gp $54.67 \pm 7.09$, nephrilin gp $42.50 \pm 7.66, P=0.017 ;{ }^{\star} P<0.05$ vs. saline-treated group

metastases in the nephrilin-treated group runs in the reverse direction of that gradient, from a $46 \%$ reduction in the accessory lobe to a $3 \%$ reduction in the superior lobe. Overall, nephrilin reduces B16MET lung metastases by $22 \%$ in all lobes combined $(P=0.017)$.

Figure 3 shows a strong and direct relationship between the accumulation of nephrilin in the various lobes and the reduction in metastases in those tissues $\left(r^{2}=0.98\right)$.

\section{DISCUSSION}

Nephrilin's efficacy in reversing the systemic effects of sepsis and burn trauma ${ }^{[9-11]}$ appears to involve pro-homeostatic modulation of the underlying immune dysfunction. In this study, we measured the distribution of nephrilin peptide across lung tissues after administration via subcutaneous bolus injection. Surprisingly, substantial preference of peptide accumulation was observed in lobes of the lung that are distal to the circulation, an environment in which we also showed a higher accumulation of succinate, a proxy for anoxia ${ }^{[23]}$. In previously published work, a similar gradient was shown for concentrations of lung elastase, fragments of which have been implicated in the inflammatory response within these tissues ${ }^{[17]}$.

Nephrilin is effective at reducing B16MET metastases to the lung in this murine model. The effectiveness of the peptide is directly proportional to the peptide's preferential accumulation in tissues of the deep lung. The potential clinical significance of this pattern of accumulation should be mentioned here: tissues of the deep lung have been associated with morbidity and mortality of respiratory distress syndromes and pneumonia, often comorbid with kidney dysfunction or sepsis, accounting for much of the deadliest burden in ICUs ${ }^{[24,25]}$. Furthermore, these deep tissues are associated with lower oxygen tensions, a feature associated with metastatic niche ${ }^{[26]}$. 


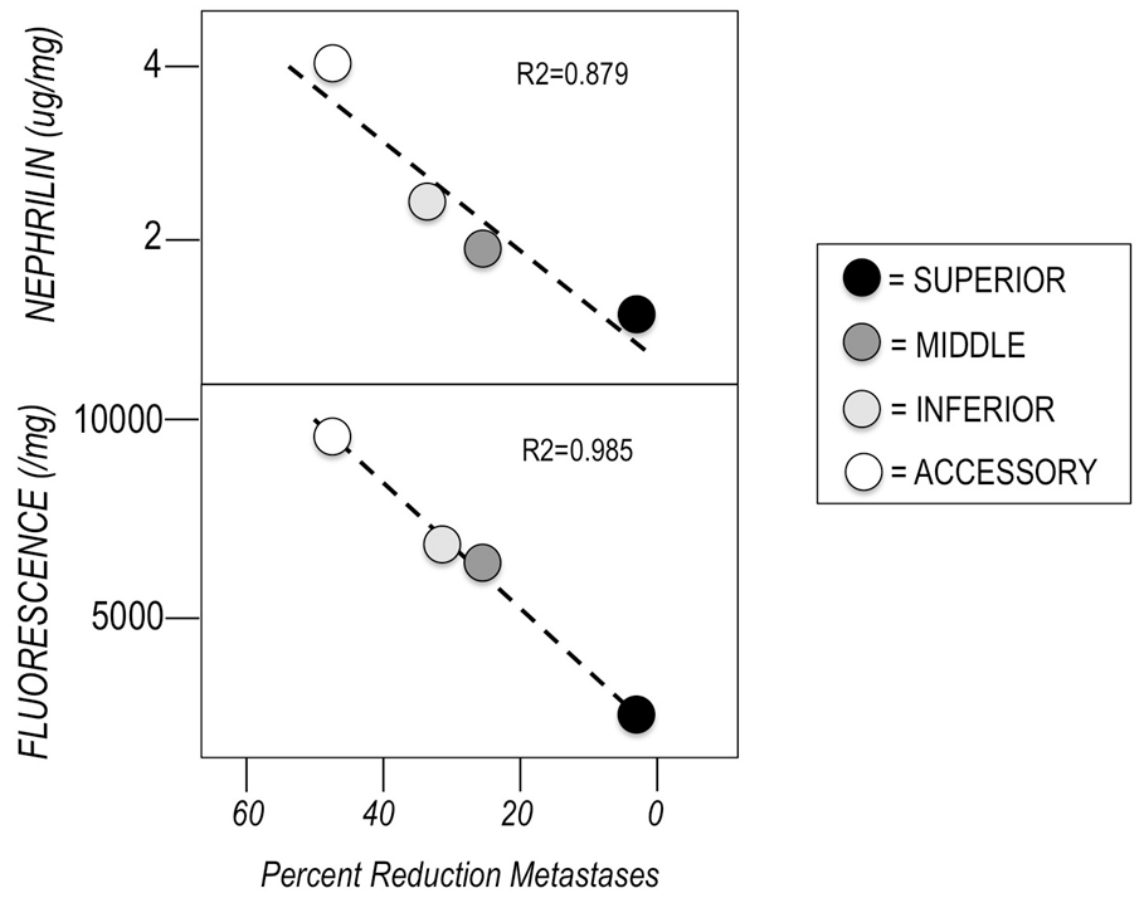

Figure 3. Biodistribution of nephrilin/tracer peptide and inhibition of metastases observed in lung lobes

Our observation that nephrilin treated animals have significantly lower plasma levels of the pro-fibrotic factors CCL12 and TIMP-1 is particularly intriguing. Fibrosis and metastasis have been linked in previous studies ${ }^{[22]}$. Whether animals marked at the genetic loci for CCL12 and TIMP-1 might show lowered altered levels of susceptibility to the metastatic spread of melanoma is worthy of exploration.

In the context of metastatic melanoma, the ability to address the formation of a metastatic niche in the deep lung is an intriguing subject for future study. But our results also raise other questions: Can the unusual lung tissue targeting seen with nephrilin peptide in this study presage its use as a vehicle for deeplung targeted treatments in general? Given the known efficacy of nephrilin peptides in models relevant to other aspects of critical care such as sepsis, burn trauma, and kidney function, the potential usefulness of this peptide for targeting treatments in ARDS is intriguing.

\section{DECLARATIONS}

\section{Authors' contributions}

Contributed to experimental work, experimental design and data discussion: Mascarenhas DD, Ravikumar P Contributed to funding the research, discussing the data and manuscript writing: Mascarenhas DD, Amento EP

\section{Availability of data and materials}

For additional data requests, please contact the corresponding author.

\section{Financial support and sponsorship}

General funds from all participating institutions contributed to the funding of this study.

\section{Conflicts of interest}

All authors declared that there are no conflicts of interest.

\section{Ethical approval and consent to participate}

All animal procedures were performed in adherence to the National Institute of Health's Guide for Care and 
Use of Laboratory Animals and approved by the Institutional Animal Care and Use Committee (IACUC) of the Molecular Medicine Research Institute.

\section{Consent for publication}

Not applicable.

\section{Copyright}

(c) The Author(s) 2020.

\section{REFERENCE}

1. Singh BK, Singh A, Mascarenhas DD. A nuclear complex of rictor and insulin receptor substrate-2 is associated with albuminuria in diabetic mice. Metab Syndr Relat Disord 2010;8:355-63.

2. Huq A, Singh B, Meeker T, Mascarenhas D. The metal-binding domain of IGFBP-3 selectively delivers therapeutic molecules into cancer cells. Anticancer Drug 2009;20:21-31.

3. Purohit M, Goldstein R, Nadler D, Mathews K, Slocum C, et al. Cognition in patients with burn injury in the inpatient rehabilitation population. Arch Phys Med Rehabil 2014;95:1342-49.

4. Jeschke MG, Pinto R, Kraft R, Nathens AB, Finnerty CC, et al. Morbidity and survival probability in burn patients in modern burn care. Crit Care Med 2015;43:808-15.

5. Jeschke MG, Pinto R, Herndon DN, Finnerty CC, Kraft R. Hypoglycemia is associated with increased postburn morbidity and mortality in pediatric patients. Crit Care Med 2014;42:1221-31.

6. Chondronikola M, Meyer WJ, Sidossis LS, Ojeda S, Huddleston J, et al. Predictors of insulin resistance in pediatric burn injury survivors 24 to 36 months postburn. J Burn Care Res 2014;35:409-15.

7. Patel P, Sallam HS, Ali A, Chandalia M, Suman O, et al. Changes in fat distribution in children following severe burn injury. Metab Syndr Relat Disord 2014;12:523-6.

8. Kraft R, Herndon DN, Finnerty CC, Shahrokhi S, Jeschke MG. Occurrence of multiorgan dysfunction in pediatric burn patients:incidence and clinical outcome. Ann Surg 2014;259:381-7.

9. Mascarenhas DD, Elayadi A, Singh BK, Prasai A, Hegde SD, et al. Nephrilin peptide modulates a neuroimmune stress response in rodent models of burn trauma and sepsis. Intl J Burns and trauma 2013;3:190-200.

10. Mascarenhas DD, Ayadi AE, Wetzel M, Prasai A, Mifflin R, et al. Effects of the nephrilin peptide on post-burn glycemic control, renal function, fat and lean body mass, and wound healing. Intl J Burns and trauma 2016;6:44-50.

11. Mascarenhas DD, Ayadi AE, Ravikumar P, Kang GJ, Langer T, et al. Positive effects of ferric iron on the systemic efficacy of nephrilin peptide in burn trauma. Scars Burn Heal 2020;6:1-8.

12. Mascarenhas DD, Herndon DN, Arany I. Epigenetic memory of oxidative stress: does nephrilin exert its protective effects via rac1? Biologics 2017;11:97-106.

13. Mascarenhas DD. Transcriptional re-programming in rat CNS two weeks after burn trauma: the impact of nephrilin treatment on the expression of oxidative stress-related genes. Scars, Burns \& Healing 2020;6:1-9.

14. Mascarenhas D, Routt S, Singh BK. Mammalian target of rapamycin complex 2 regulates inflammatory response to stress. Inflamm Res 2012;61:1395-404.

15. Quail DF, Joyce JA. Microenvironmental regulation of tumor progression and metastasis. Nat Med 2013;19:1423-37.

16. Liu Y, Cao X. Characteristics and significance of the pre-metastatic niche. Cancer Cell 2016;30:668-81.

17. Young SM, Liu S, Joshi R, Batie MR, Kofron M, et al. Localization and stretch-dependence of lung elastase activity in development and compensatory growth. J Appl Physiol 2015;118:921-31.

18. Estenssoro E, Dubin A. Acute respiratory distress syndrome. Medicina (B Aires) 2016;76:235-41.

19. Gudaviciene D, Rimdeika R, Adamonis K. Influence of Enteral Nutrition on the Frequency of Complications in Case of Major Burns. Medicina (Kaunas) 2004;40:957-61.

20. Shi H, Zhang J, Han X, Li H, Xie M, et al. Recruited monocytic myeloid-derived suppressor cells promote the arrest of tumor cells in the premetastatic niche through an il-1 $\beta$-mediated increase in e-selectin expression. Int J Cancer 2017;140:1370-83.

21. Guo J, Guan Q, Liu X, Wang H, Gleave ME, et al. Relationship of clusterin with renal inflammation and fibrosis after the recovery phase of ischemia-reperfusion injury. BMC Nephrol 2016;17:133.

22. Lebrun A, Lo Re S, Chantry M, Izquierdo Carerra X, Uwambayinema F, et al. CCR2 monocytic myeloid-derived suppressor cells (M-MDSCs) inhibit collagen degradation and promote lung fibrosis by producing transforming growth factor- $\beta 1$. J Pathol 2017;243:320-330.

23. Jiang S, Yan W. Succinate in the cancer-immune cycle. Cancer Lett 2017;390:45-7.

24. Thakkar RK, Weiss SL, Fitzgerald JC, Keele L, Thomas NJ, et al. Risk factors for mortality in pediatric postsurgical versus medical severe sepsis. J Surg Res 2019;242:100-10.

25. Fitzgerald JC, Ross ME, Thomas NJ, Weiss SL, Balamuth F, et al. Risk factors and inpatient outcomes associated with acute kidney injury at pediatric severe sepsis presentation. Pediatr Nephrol 2018;33:1781-90.

26. Philip B, Ito K, Moreno-Sánchez R, Ralph SJ. HIF expression and the role of hypoxic microenvironments within primary tumours as protective sites driving cancer stem cell renewal and metastatic progression. Carcinogenesis 2013;34:1699-707. 